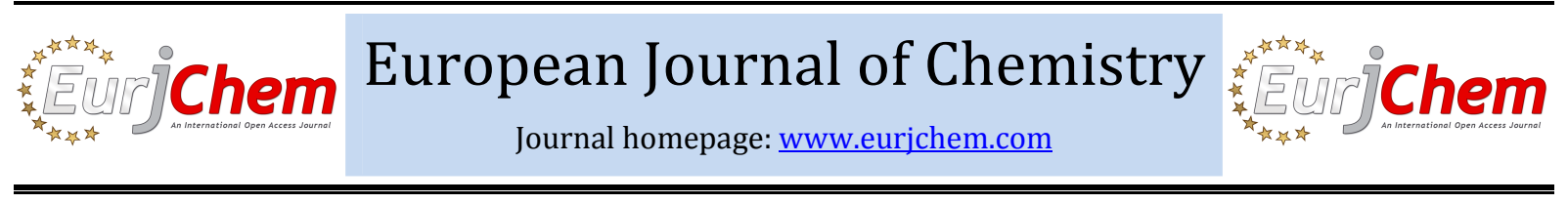

\title{
Synthesis and characterization of novel pyrazolone derivatives
}

\author{
Magda Ismail Marzouk a,*, Galal Hosni Sayed a, \\ Mohamed Said Abd ElHalim ${ }^{\mathrm{b}}$ and Salma Yehia Mansour a \\ a Heterocyclic Synthetic Laboratory, Chemistry Department, Faculty of Science, Ain Shams University, Abbassia 11566, Cairo, Egypt \\ ${ }^{\mathrm{b}}$ Heterocyclic Synthetic Laboratory, Chemistry Department, Faculty of Engineering, Ain Shams University, Abbassia 11566, Cairo, Egypt \\ *Corresponding author at: Heterocyclic Synthetic Laboratory, Chemistry Department, Faculty of Science, Ain Shams University, Abbassia 11566, Cairo, Egypt. \\ Tel.: +2.0122.3309974. Fax: +2.02.24662917. E-mail address: magda marzouk@sci.asu.edu.eg (M. Marzouk).
}

ARTICLE INFORMATION

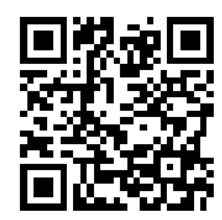

DOI: $10.5155 /$ eurjchem.5.1.24-32.870

Received: 08 July 2013

Received in revised form: 26 August 2013

Accepted: 26 August 2013

Online: 31 March 2014

\section{KEYWORDS}

Pyrazolone

Malononitrile

Azo compounds

Pyrazolo pyrimidine

Microwave irradiation

$\alpha, \beta$-Unsaturated ketone

\section{Introduction}

Pyrazolone is a five membered lactam ring [1] containing two nitrogen atoms and one ketonic group in its structure. During the discovery of pyrazolone, they were only known as NSAIDs [2] but, since year 2000, they play versatile role in several complications like cerebral ischemia and cardiovascular diseases [3]. The chemistry of pyrazolone has gained increasing attention due to its diverse pharmacological properties [4] such as cytotoxic [5,6], anti-inflammatory [7], antimicrobial [8,9], antioxidant [10], antiviral [11], oral hypoglycemic activity [12] and analgesic [13,14]. Pyrazolones are believed to be involved in various biochemical and physiological reactions and thus scientific research programs are continually improvising synthetic techniques to prepare numerous pyrazolone derivatives. For the last decade a lot of work has been going on the pyrazolone nucleus.

Scientists have developed large number of new compounds related to this moiety and screened them for their different pharmacological activities to get a molecule of desired pharmacological activity. Although earlier many of pyrazolone derivatives were associated with adverse effects such as agranulocytosis, skin rashes and blood dyscariasis, etc.

\section{Experimental}

\subsection{Instrumentation}

All melting points were measured on a Gallenkamp melting point apparatus and are uncorrected. All microwave reactions were carried out in a domestic microwave oven (Galanza 950). The infrared spectra were recorded using potassium bromide disks on a Pye Unicam SP-3-300 infrared spectrophotometer. ${ }^{1} \mathrm{H}$ NMR spectra were run at $300 \mathrm{MHz}$, on a Varian Mercury VX300 NMR spectrometer, using TMS as internal standard in deuterated chloroform or deuterated dimethyl sulphoxide. Chemical shifts are quoted $\delta$ in ppm. The mass spectra were recorded on Shimadzu GCMS-QP-1000EX mass spectrometers at $70 \mathrm{eV}$. All the spectral measurements as well as elemental analyses were carried out at the Micro Analytical Center of Cairo University, Egypt, and the Main Defence Chemical Laboratory 2. The antimicrobial activities were carried out at Egyptian Petroleum Research Institute, Fermentation Biotechnology Lab. All the chemical reactions are monitored by TLC. 


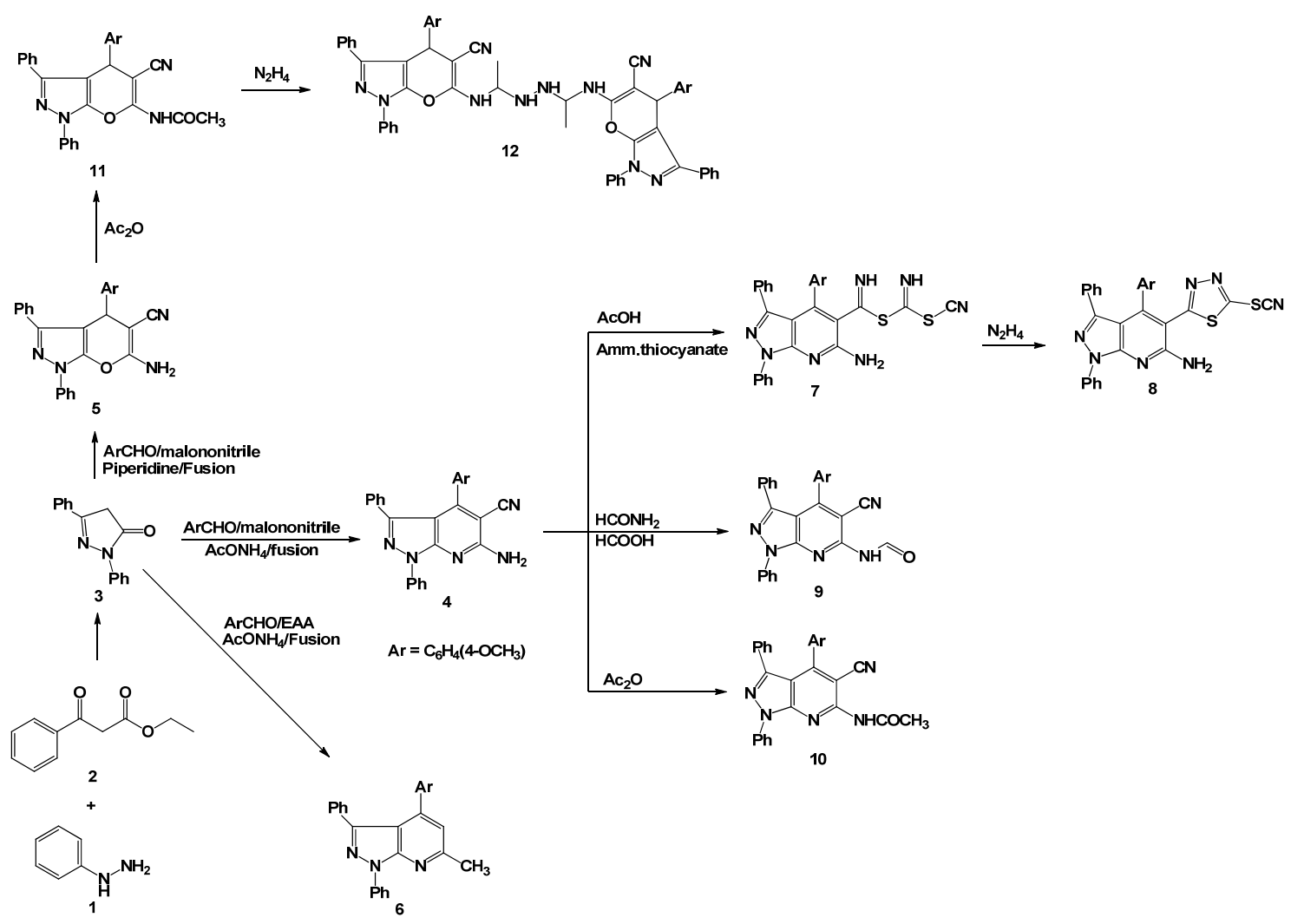

Scheme 1

\subsection{Synthesis}

\subsubsection{1,3-Diphenyl-1H-pyrazol-5(4H)-one (3)}

A mixture of phenyl hydrazine (1) $(10.8 \mathrm{~mL}, 0.1 \mathrm{~mol})$, ethyl benzoylacetate (2) $(19.2 \mathrm{~mL}, 0.1 \mathrm{~mol})$ in acetic acid $(50 \mathrm{~mL})$ was refluxed for $4 \mathrm{~h}$, and left to cool. The separated solid was filtered off, washed with ethanol, dried and recrystallized from acetic acid. Color: Yellow. Yield: 90\%. M.p.: 138-140 ${ }^{\circ} \mathrm{C}$ (Lit. [15], $135{ }^{\circ} \mathrm{C}$ ). FT-IR (KBr, v, cm-1): 3055 (CH aromatic), 2954, 2911 (CH aliphatic), 1706 (C=0). ${ }^{1} \mathrm{H}$ NMR (300 MHz, $\mathrm{CDCl}_{3}, \delta$, ppm): 7.94 (d, 2H, ArH), 7.80 (d, 2H, ArH), 7.34-7.53 (m, 4H, ArH), 7.42 (t, 2H, $\mathrm{ArH}$ ), 3.87 (s, 2H, $\mathrm{CH}_{2}$ ). MS (EI, $m / z$ (\%)): 236 $\left(\mathrm{M}^{+*}, 100\right), 194$ (10.51), 105 (29.76), 103 (63.39), 91 (53.48), 78 (7.19), 77 (84.85). Anal. calcd. for $\mathrm{C}_{15} \mathrm{H}_{12} \mathrm{~N}_{2} \mathrm{O}$ : C, 76.25; H, 5.12; N, 11.86. Found: C, 76.36; H, 5.14; N, $12.01 \%$.

\subsubsection{6-Amino-4-(4-methoxyphenyl)-1,3-diphenyl-1H- pyrazolo[3,4-b]pyridine-5-carbonitrile (4)}

A mixture of pyrazolone $3(2.36 \mathrm{~g}, 0.01 \mathrm{~mol})$, malononitrile $(0.66 \mathrm{~g}, 0.01 \mathrm{~mol})$, ammonium acetate $(2.31 \mathrm{~g}, 0.03 \mathrm{~mol})$, and $p$ anisaldehyde $(1.36 \mathrm{~mL}, 0.01 \mathrm{~mol})$ was fused for $15 \mathrm{~h}$ at $140-170$ ${ }^{\circ} \mathrm{C}$, the reaction mixture was poured onto water, to give the solid product 4 which was washed with water $(3 \times 30 \mathrm{~mL})$, dried, and recrystallized from benzene:ethanol (2:1) (Scheme 1). Color: Brown. Yield: 86.3 \%. M.p.: $248-250^{\circ} \mathrm{C}$. FT-IR (KBr, v, $\left.\mathrm{cm}^{-1}\right)$ : 3451, $3338\left(\mathrm{NH}_{2}\right), 2202(\mathrm{C} \equiv \mathrm{N}), 1604(\mathrm{C}=\mathrm{N}) .{ }^{1} \mathrm{H}$ NMR (300 MHz, DMSO, $\delta, p p m), \delta: 7.06-7.14(\mathrm{~m}, 14 \mathrm{H}, \mathrm{ArH}), 5.02$ (s, $2 \mathrm{H}, \mathrm{NH}_{2} \mathrm{D}_{2} \mathrm{O}$ exchangeable), $3.82\left(\mathrm{~s}, 3 \mathrm{H}, \mathrm{OCH}_{3}\right)$. MS (EI, $m / z$ (\%)): $417\left(\mathrm{M}^{+}, 0.80\right), 330$ (74.22), 287 (24.48), 121 (100), 77 (32.72). Anal. calcd. for $\mathrm{C}_{26} \mathrm{H}_{19} \mathrm{~N}_{5} \mathrm{O}$ : C, 74.80; H, 4.59; N, 16.78 . Found: C, 74.95; H, 4.72; N, $16.68 \%$.

\subsubsection{6-Amino-4-(4-methoxyphenyl)-1,3-diphenyl-1,4- dihydropyrano[2,3-c]pyrazole-5-carbonitrile (5)}

Method A: A mixture of pyrazolone $3(2.36 \mathrm{~g}, 0.01 \mathrm{~mol})$, malononitrile $(0.66 \mathrm{~g}, 0.01 \mathrm{~mol})$, and $p$-anisaldehyde $(1.36 \mathrm{~mL}$, $0.01 \mathrm{~mol}$ ) in presence of $1 \mathrm{~mL}$ piperidine was fused for $15 \mathrm{~h}$ at $140{ }^{\circ} \mathrm{C}$. After cooling, the solid product was washed with ethanol, filtered off, dried, and crystallized from benzene: ethanol (2:1) (Scheme 1). Color: Brown. Yield: 14 \%. M.p.: 288$290^{\circ} \mathrm{C}$. FT-IR (KBr, v, $\left.\mathrm{cm}^{-1}\right): 3400,3376\left(\mathrm{NH}_{2}\right), 2206(\mathrm{C} \equiv \mathrm{N})$, $1600(\mathrm{C}=\mathrm{N}) .{ }^{1} \mathrm{H}$ NMR $(300 \mathrm{MHz}, \mathrm{DMSO}, \delta, \mathrm{ppm}): 6.97-7.44(\mathrm{~m}$, $16 \mathrm{H}, 14 \mathrm{ArH}, \mathrm{NH}_{2}$ ), 3.83 (s, 1H, benzilic), 3.70 (s, 3H, $\mathrm{OCH}_{3}$ ). MS (EI, $m / z(\%)): 420$ (6.83, M+), 408 (27.95), 360 (22.67), 247 (10.25), 95 (23.91), 55 (100). Anal. calcd. for $\mathrm{C}_{26} \mathrm{H}_{20} \mathrm{~N}_{4} \mathrm{O}_{2}$ : C, 74.27; H, 4.79; N, 13.33. Found: C, 74.41; H, 4.98; N, $13.54 \%$.

Method B: A mixture of pyrazolone $3(2.36 \mathrm{~g}, 0.01 \mathrm{~mol})$, malononitrile $(0.66 \mathrm{~g}, 0.01 \mathrm{~mol})$, and $p$-anisaldehyde $(1.36 \mathrm{~mL}$, $0.01 \mathrm{~mol}$ ) in presence of $1 \mathrm{~mL}$ piperidine was heated under microwave irradiation for $2 \mathrm{~min}$, at power 180 Watt. After cooling, the solid product was washed with ethanol, filtered off, dried, and crystallized from benzene:ethanol (2:1).

\subsubsection{4-(4-Methoxyphenyl)-6-methyl-1,3-diphenyl-1H- pyrazolo[3,4-b]pyridine (6)}

A mixture of pyrazolone $3(2.36 \mathrm{~g}, 0.01 \mathrm{~mol})$, ethyl acetoacetate $(2.94 \mathrm{~mL}, 0.01 \mathrm{~mol}$,$) , ammonium acetate (2.31 \mathrm{~g}$, $0.03 \mathrm{~mol})$, and $p$-anisaldehyde $(1.36 \mathrm{~mL}, 0.01 \mathrm{~mol})$ was fused for $15 \mathrm{~h}$ at $140{ }^{\circ} \mathrm{C}$. After cooling, the solid product was washed with water, filtered off, dried, and crystallized from benzene:petroleum ether $\left(60-80{ }^{\circ} \mathrm{C}\right)(2: 1)$ (Scheme 1). Color: Brown. Yield: 50 \%. M.p.: 246-248 ${ }^{\circ} \mathrm{C}$. FT-IR (KBr, v, cm-1): 3058 (CH aromatic), 2958 (CH aliphatic), $1635(\mathrm{C}=\mathrm{N}), 1601(\mathrm{C}=\mathrm{C})$. 
${ }^{1} \mathrm{H}$ NMR (300 MHz, DMSO, $\left.\delta, \mathrm{ppm}\right): 6.88-8.11(\mathrm{~m}, 15 \mathrm{H}$, $\mathrm{ArH}), 3.81\left(\mathrm{~s}, 3 \mathrm{H}, \mathrm{OCH}_{3}\right), 3.30$ (s, $\left.3 \mathrm{H}, \mathrm{CH}_{3}\right)$. MS (EI, $\left.m / z(\%)\right)$ : $391\left(21.7, \mathrm{M}^{+}\right), 354$ (100), 353 (82.6), 188 (26.1), 77 (95.7), 68 (13.0). Anal. calcd. for $\mathrm{C}_{26} \mathrm{H}_{21} \mathrm{~N}_{3} \mathrm{O}$ : C, 79.77; H, 5.41; N, 10.73 . Found: C, 79.91; H, 5.60; N, $10.96 \%$.

\subsection{5. (6-Amino-4-(4-methoxyphenyl)-1,3-diphenyl-1H- pyrazolo[3,4-b]pyridin-5-yl)(iminomethyl) thiocyanatomethanethioimidate (7)}

A mixture of pyrazolopyridine-5-carbonitrile 4 (4.17g, $0.01 \mathrm{~mol})$, ammonium thiocyanate $(2.28 \mathrm{~g}, 0.03 \mathrm{~mol})$, in acetic acid $(10 \mathrm{~mL})$ was refluxed for $10 \mathrm{~h}$, the reaction mixture was poured onto water, filtered off, washed with water, dried and recrystallized from ethanol (Scheme 1). Color: Brown. Yield: 92 \%. M.p.: 222-224 ${ }^{\circ}$ C. FT-IR (KBr, v, $\left.\mathrm{cm}^{-1}\right): 3457,3338\left(\mathrm{NH}, \mathrm{NH}_{2}\right)$, $2207(\mathrm{C} \equiv \mathrm{N}), 1602(\mathrm{C}=\mathrm{N}) .{ }^{1} \mathrm{H}$ NMR $\left(300 \mathrm{MHz}, \mathrm{CDCl}_{3}, \delta, \mathrm{ppm}\right)$ : 6.94-7.95(m, 14H, ArH), $5.69\left(\mathrm{~s}, 4 \mathrm{H}, 2 \mathrm{NH}, \mathrm{NH}_{2} \mathrm{D}_{2} \mathrm{O}\right.$ exchangeable), $3.78\left(\mathrm{~s}, 3 \mathrm{H}, \mathrm{OCH}_{3}\right)$. MS (EI, $\left.\mathrm{m} / z(\%)\right)$ : 535 (20.0, $\mathrm{M}^{+}$) 376 (16.7), 195 (16.7), 103 (100), 104 (46.7), 79 (26.7), 78 (36.7), 77(96.7). Anal. calcd. for $\mathrm{C}_{28} \mathrm{H}_{21} \mathrm{~N}_{7} \mathrm{OS}_{2}$ : C, 62.78; H, 3.95; N, 18.30; S, 11.97. Found: C, 62.92; H, 4.15; N, 12.13; S, $11.94 \%$.

\subsubsection{4-(4-Methoxyphenyl)-1,3-diphenyl-5-(5-thiocyanato-} 1,3,4-thiadiazol-2-yl)-1H-pyrazolo[3,4-b]pyridin-6-amine (8)

A mixture of (iminomethyl) thiocyanatomethanethioimidate 7 (5.35 g, $0.01 \mathrm{~mol})$, hydrazine hydrate $(0.5 \mathrm{~mL}$, $0.01 \mathrm{~mol})$, in absolute ethanol $(20 \mathrm{~mL})$ was refluxed for $2 \mathrm{~h}$, the reaction mixture was concentrated, and the separated solid was filtered off, dried and crystallized from ethanol:benzene (1:1) (Scheme 1). Color: Dark brown. Yield: 35 \%. M.p.: 225-227 ${ }^{\circ} \mathrm{C}$. FT-IR (KBr, v, cm$\left.{ }^{-1}\right): 3324\left(\mathrm{NH}_{2}\right), 1602(\mathrm{C}=\mathrm{N}), 1252(\mathrm{C}=\mathrm{C})$. ${ }_{1}^{1} \mathrm{H}$ NMR $\left(300 \mathrm{MHz}, \mathrm{CDCl}_{3}, \delta, \mathrm{ppm}\right): 7.02-8.05(\mathrm{~m}, 14 \mathrm{H}, \mathrm{ArH})$, $5.63\left(\mathrm{~s}, 2 \mathrm{H}, \mathrm{NH}_{2} \mathrm{D}_{2} \mathrm{O}\right.$ exchangeable), $3.87\left(\mathrm{~s}, 3 \mathrm{H}, \mathrm{OCH}_{3}\right)$. MS (EI, $\mathrm{m} / \mathrm{z}(\%)$ ): 533 (0.01, $\mathrm{M}^{+}$), 516 (14.3), 210 (17.1), 119 (45.7), 93 (25.7), 60 (25.7), 58 (17.1), 57 (100). Anal. calcd. for $\mathrm{C}_{28} \mathrm{H}_{19} \mathrm{~N}_{7} \mathrm{OS}_{2}$ : C, 63.02; H, 3.59; N, 18.37; S, 12.02. Found: C, 63.28; H, 4.13; N, 19.02; S, $12.25 \%$.

\subsection{7. $\mathrm{N}$-(5-cyano-4-(4-methoxyphenyl)-1,3-diphenyl-1H- pyrazolo[3,4-b]pyridin-6-yl)formamide (9)}

A mixture of pyrazolopyridine-5-carbonitrile 4 (4.17g, $0.01 \mathrm{~mol})$, formic acid $(5 \mathrm{~mL})$, formamide $(10 \mathrm{~mL})$, and dimethyl formamide $(5 \mathrm{~mL})$ was refluxed for $12 \mathrm{~h}$, the reaction mixture was poured onto water to give solid which was filtered off, washed with water, dried and recrystallized from benzene (Scheme 1). Color: Green. Yield: 76.4 \%. M.p.: 230-232 ${ }^{\circ} \mathrm{C}$. FT-IR $\left(\mathrm{KBr}, v, \mathrm{~cm}^{-1}\right): 3428(\mathrm{NH}), 2208(\mathrm{C} \equiv \mathrm{N}), 1670(\mathrm{C}=0), 1604$ (C=N). ${ }^{1} \mathrm{H}$ NMR $\left(300 \mathrm{MHz}, \mathrm{CDCl}_{3}, \delta, \mathrm{ppm}\right): 7.02-8.20(\mathrm{~m}, 15 \mathrm{H}$, $14 \mathrm{ArH},-\mathrm{CH}), 5.73\left(\mathrm{~s}, 1 \mathrm{H}, \mathrm{NH} \mathrm{D}_{2} \mathrm{O}\right.$ exchangeable), $3.87(\mathrm{~s}, 3 \mathrm{H}$, $\mathrm{OCH}_{3}$ ). MS (EI, $\left.m / z(\%)\right): 445\left(0.01, \mathrm{M}^{+}\right), 386$ (21.4), 354 (21.4), 238 (16.7), 194 (26.2), 119 (23.8), 106 (23.8), 93 (31.0), 77 (100). Anal. calcd. for $\mathrm{C}_{27} \mathrm{H}_{19} \mathrm{~N}_{5} \mathrm{O}_{2}$ : C, 72.80; $\mathrm{H}, 4.30 ; \mathrm{N}, 15.72$. Found: C, 73.13; H, 4.70; N, $15.90 \%$.

\subsubsection{General procedure for compounds 10 and 11}

A mixture of amino carbonitrile 4 and/or $5(0.01 \mathrm{~mol})$ and acetic anhydride $(20 \mathrm{~mL})$ was refluxed for $5 \mathrm{~h}$, the reaction mixture was poured onto water, the separated solid was filtered off, washed with water, dried and recrystallized from benzene:ethanol (2:1) (Scheme 2).

N-(5-cyano-4-(4-methoxyphenyl)-1,3-diphenyl-1H-pyrazolo [3,4-b]pyridin-6-yl)acetamide (10): Color: Brown. Yield: 85.45 \%. M.p.:234-236 ${ }^{\circ} \mathrm{C}$. FT-IR (KBr,v, cm$\left.{ }^{-1}\right): 3436(\mathrm{NH}), 2216$ $(\mathrm{C} \equiv \mathrm{N}), 1712(\mathrm{C}=0) .{ }^{1} \mathrm{H}$ NMR $\left(300 \mathrm{MHz}, \mathrm{CDCl}_{3}, \delta, \mathrm{ppm}\right): 7.01-$ $8.10(\mathrm{~m}, 15 \mathrm{H}, 14 \mathrm{ArH}, \mathrm{NH}), 3.92\left(\mathrm{~s}, 3 \mathrm{H}, \mathrm{OCH}_{3}\right), 1.26(\mathrm{~s}, 3 \mathrm{H}$, $\mathrm{COCH}_{3}$ ). MS (EI, $\left.m / z(\%)\right): 459\left(16.7 \%, \mathrm{M}^{+}\right), 355$ (19.4), 279
(16.7), 135 (27.8), 121 (50.0), 119 (22.2), 93 (22.2), 78 (16.7), 77 (100). Anal. calcd. for $\mathrm{C}_{28} \mathrm{H}_{21} \mathrm{~N}_{5} \mathrm{O}_{2}$ : C, 73.19; $\mathrm{H}, 4.61 ; \mathrm{N}, 15.24$. Found: C, 73.17; H, 4.50; N, $15.36 \%$.

$N$-(5-cyano-4-(4-methoxyphenyl)-1,3-diphenyl-1,4-dihydro pyrano[2,3-c]pyrazol-6-yl)acetamide (11): Color: Dark brown. Yield: 55.88 \%. M.p.: $>300^{\circ} \mathrm{C}$. FT-IR (KBr, v, cm-1): $3421(\mathrm{NH})$, $2211(\mathrm{C} \equiv \mathrm{N}), 1714(\mathrm{C}=0), 1598(\mathrm{C}=\mathrm{N}) .{ }^{1} \mathrm{H}$ NMR $(300 \mathrm{MHz}$, $\left.\mathrm{CDCl}_{3}, \delta, \mathrm{ppm}\right): 6.47-7.90(\mathrm{~m}, 15 \mathrm{H}, 14 \mathrm{ArH}, \mathrm{NH}), 3.94(\mathrm{~s}, 1 \mathrm{H}$, benzilic), $3.78\left(\mathrm{~s}, 3 \mathrm{H}, \mathrm{OCH}_{3}\right), 2.07\left(\mathrm{~s}, 3 \mathrm{H}, \mathrm{COCH}_{3}\right)$. MS (EI, $\mathrm{m} / z$ (\%)): 431 (7.8, [M - $\left.\left.\mathrm{CH}_{3} \mathrm{O}\right]^{+}\right), 236$ (19.6), 126 (13.7), 84 (25.5), 70 (27.5), 64 (100), 55 (23.5), 53 (37.3). Anal. calcd. for $\mathrm{C}_{28} \mathrm{H}_{22} \mathrm{~N}_{4} \mathrm{O}_{3}: \mathrm{C}, 72.71 ; \mathrm{H}, 4.79 ; \mathrm{N}, 12.11$. Found: $\mathrm{C}, 72.65 ; \mathrm{H}, 4.58$; $\mathrm{N}, 12.90 \%$.

\subsubsection{6,6'-(1,1'(Hydrazine-1,2-diylbis(ethane-1,1-diyl)) bis(azanediyl))bis(4-(4-methoxyphenyl)-1,3-diphenyl-1,4- dihydropyrano[2,3-c]pyrazole-5-carbonitrile) (12)}

A mixture of pyrazoloacetamide 11 (4.62 g, $0.01 \mathrm{~mol})$ hydrazine hydrate $(0.5 \mathrm{~mL}, 0.01 \mathrm{~mol})$ in absolute ethanol $(50 \mathrm{~mL})$ was refluxed for $2 \mathrm{~h}$, the reaction mixture was poured onto ice: $\mathrm{HCl}$ (1:3). The solid that separated was filtered off, washed with water, dried and recrystallized from benzene:ethanol (2:1) (Scheme 1). Color: Pale brown. Yield: 22 \%. M.p.: 280-282 ${ }^{\circ} \mathrm{C}$. FT-IR (KBr, v, cm-1): $3336(\mathrm{NH}), 2207$ $(\mathrm{C} \equiv \mathrm{N}), 1600(\mathrm{C}=\mathrm{N}) .{ }^{1} \mathrm{H}$ NMR $\left(300 \mathrm{MHz}, \mathrm{CDCl}_{3}, \delta, \mathrm{ppm}\right): 6.83-$ $7.79(\mathrm{~m}, 28 \mathrm{H}, \mathrm{ArH}), 3.83$ (s, 2H, benzilic), $3.69(\mathrm{~m}, 2 \mathrm{H}, 2 \mathrm{CHNH})$, $3.49\left(\mathrm{~s}, 6 \mathrm{H}, 2 \mathrm{CH}_{3}\right), 2.73\left(\mathrm{~s}, 4 \mathrm{H}, 4 \mathrm{NH} \mathrm{D}_{2} \mathrm{O}\right.$ exchangeable), 1.04(d, $\left.6 \mathrm{H}, 2 \mathrm{CH}_{3}\right)$. MS (EI, $m / z(\%)$ ): $926(18.8, \mathrm{M}+2), 319$ (15.6), 234 (15.6), 128 (12.5), 112 (18.8), 84 (40.6), 78 (40.6), 77 (62.5), 60 (46.9), 58 (56.3), 56 (75.0), 55 (100). Anal. calcd. for $\mathrm{C}_{56} \mathrm{H}_{48} \mathrm{~N}_{10} \mathrm{O}_{4}$ : C, $72.71 ; \mathrm{H}, 5.23 ; \mathrm{N}, 15.14$. Found: $\mathrm{C}, 72.53 ; \mathrm{H}$, 5.34; N, $15.20 \%$.

\subsubsection{4-(2,3,4,5,6-Pentahydroxyhexylidene)-1,3-diphenyl- 1H-pyrazol-5(4H)-one (13)}

A mixture of pyrazolone $3(2.36 \mathrm{~g}, 0.01 \mathrm{~mol})$, glucose $(2.3 \mathrm{~g}$, $0.01 \mathrm{~mol})$ in $\mathrm{H}_{2} \mathrm{O}(1 \mathrm{~mL})$, ethanol $(5 \mathrm{~mL})$, and DMF $(5 \mathrm{~mL})$ was heated on water bath for $4 \mathrm{~h}$, the solid that separated after cooling and evaporating the solvent was filtered off, washed with water $(30 \mathrm{~mL})$, dried and crystallized from benzene:ethanol (3:1) (Scheme 2). Color: Green. Yield: $95 \%$. M.p.: $190-192{ }^{\circ} \mathrm{C}$. FT-IR (KBr, v, cm$\left.{ }^{-1}\right): 3380(\mathrm{OH}), 3066(\mathrm{CH}$ aromatic), 2930 (CH aliphatic), 1696 (C=0). ${ }^{1} \mathrm{H}$ NMR (300 MHz, DMSO, $\delta, \mathrm{ppm}):$ 7.77-7.93 (m, 4H, ArH), 7.49-7.62 (m, 5H, $5 \mathrm{ArH}), 7.30-7.37(\mathrm{~m}, 2 \mathrm{H}, 1 \mathrm{ArH},=\mathrm{CH}), 4.60,4.92,5.96,6.07(\mathrm{~s}$, $5 \mathrm{H}, 5 \mathrm{OH}, \mathrm{D}_{2} \mathrm{O}$ exchangeable), 4.03-4.16 $(\mathrm{m}, 1 \mathrm{H},=\mathrm{CHCHOH})$, 3.77-3.86 (m, 2H, $\left.\mathrm{CH}_{2} \mathrm{OH}\right), 3.61-3.67(\mathrm{~m}, 3 \mathrm{H}$, glucose moiety). MS (EI, $m / z$ (\%)): 398 (0.06, M+), 24.7 (20.12), 236 (13.36), 91 (100), 92 (16.16), 76 (14.01). Anal. calcd. for $\mathrm{C}_{21} \mathrm{H}_{22} \mathrm{~N}_{2} \mathrm{O}_{6}$ : C, 63.31; H, 5.57; N, 7.03. Found: C, 63.50; H, 5.77; N, $7.15 \%$.

\subsubsection{5-Oxo-N,1,3-triphenyl-4,5-dihydro-1H- pyrazole-4-carbothioamide (15)}

A mixture of pyrazolone $3(2.36 \mathrm{~g}, 0.01 \mathrm{~mol})$ and phenyl isothiocyanate $(0.6 \mathrm{~mL}, 0.01 \mathrm{~mol})$ in dry acetone $(50 \mathrm{~mL})$ in the presence of piperidine $(0.5 \mathrm{~mL})$ was heated on water bath for $4 \mathrm{~h}$. The separated solid after pouring onto ic:eHCl (1:2) was filtered off, washed with water, dried and crystallized from ethanol (Scheme 2). Color: Yellow. Yield: 25.3\%. M.p.: 180-182 ${ }^{\circ} \mathrm{C}$. FT-IR (KBr, v, cm${ }^{-1}$ ): $3063(\mathrm{NH}), 3034$ (CH aromatic), 2912 (CH aliphatic), $1711(\mathrm{C}=0) .{ }^{1} \mathrm{H}$ NMR $\left(300 \mathrm{MHz}, \mathrm{CDCl}_{3}, \delta, \mathrm{ppm}\right)$ : $8.64\left(\mathrm{~s}, 1 \mathrm{H}, \mathrm{OH}, \mathrm{D}_{2} \mathrm{O}\right.$ exchangeable), 6.91-8.08 (m, 15H, ArH), $1.60\left(\mathrm{~s}, 1 \mathrm{H}, \mathrm{NH}, \mathrm{D}_{2} \mathrm{O}\right.$ exchangeable). MS (EI, $\left.\mathrm{m} / z(\%)\right): 373$ $\left(3.33, \mathrm{M}^{+2}\right), 371\left(40.86, \mathrm{M}^{+}\right), 279$ (22.20), 278 (100), 93 (21.48), 77 (56.96). Anal. calcd. for $\mathrm{C}_{22} \mathrm{H}_{17} \mathrm{~N}_{3} \mathrm{OS}$ : C, 71.14; $\mathrm{H}, 4.61 ; \mathrm{N}$, 11.31; S, 8.63. Found: C, 70.83; H, 5.32; N, 11.32; S $8.71 \%$. 


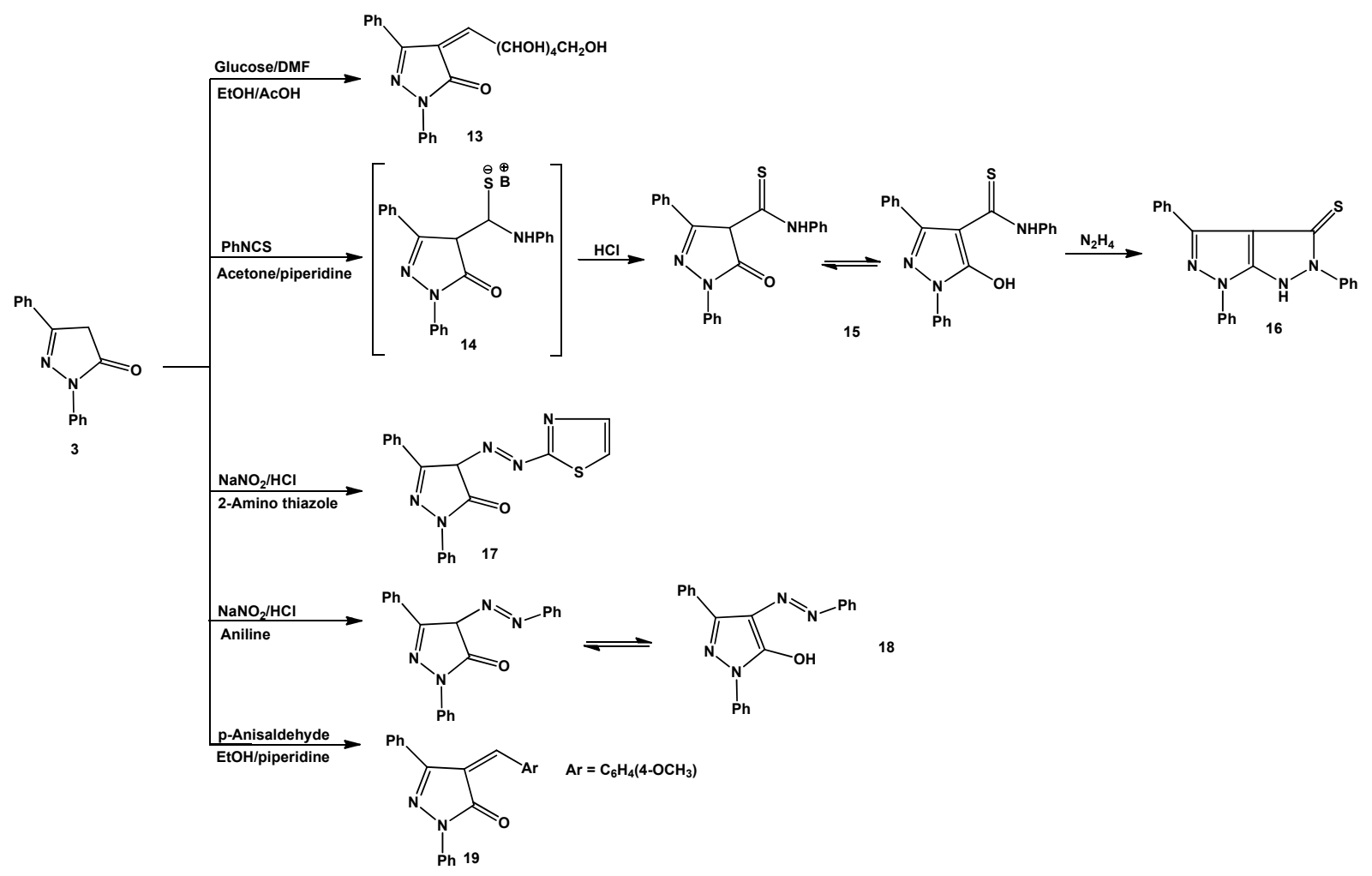

Scheme 2

2.2.12. 2,4,6-Triphenyl-1,2-dihydropyrazolo[3,4-c]pyrazole3-(6H)-thione (16)

A mixture of pyrazolidine-4-carbothioamide 15 (3.71 g, $0.01 \mathrm{~mol})$ hydrazine hydrate $(0.5 \mathrm{~mL}, 0.01 \mathrm{~mol})$ in absolute ethanol $(50 \mathrm{~mL})$ was refluxed for $10 \mathrm{~h}$. After cooling the reaction mixture was poured onto ice: $\mathrm{HCl}(1: 3)$, the separated solid was filtered off, washed with water, dried and recrystallized from benzene (Scheme 2). Color: White. Yield: 65 \%. M.p.: 235-237 ${ }^{\circ} \mathrm{C}$. FT-IR (KBr, v, cm-1): $3011(\mathrm{NH}), 1597$ $(\mathrm{C}=\mathrm{N}), 1454$ (NCS). ${ }^{1} \mathrm{H}$ NMR (300 MHz, DMSO, $\left.\delta, \mathrm{ppm}\right):$ 7.23$7.91(\mathrm{~m}, 16 \mathrm{H}, 15 \mathrm{ArH}, \mathrm{NH})$. MS (EI, $m / z(\%)): 368\left(8.00, \mathrm{M}^{+}\right)$, 260 (100), 108 (12.0), 97 (26.0), 85 (32.0), 83 (30.0), 68 (24.0), 55 (46.0). Anal. calcd. for $\mathrm{C}_{22} \mathrm{H}_{16} \mathrm{~N}_{4} \mathrm{~S}$ : C, 71.71; H, 4.38; N, 15.21; S, 8.70. Found: C, 71.92; H, 4.51; N, 15.34; S, $8.90 \%$.

\subsubsection{1,3-Diphenyl-4-(thiazol-2-yldiazenyl)-1H-pyrazol- 5(4H)-one (17)}

2-Aminothiazole $(0.1 \mathrm{~g}, 0.001 \mathrm{~mol})$ was dissolved in conc. $\mathrm{HCl}(5 \mathrm{~mL})$ and the solution was then cooled to $0-5^{\circ} \mathrm{C}$. Sodium nitrite $(0.069 \mathrm{~g}, 0.001 \mathrm{~mol})$ in water $(3 \mathrm{~mL})$ was then added to this solution dropwise with vigorous stirring during about $1 \mathrm{~h}$, while cooling at $0-5{ }^{\circ} \mathrm{C}$. The clear diazonium salt solution was then added dropwise to a well cooled $\left(0-5{ }^{\circ} \mathrm{C}\right)$ and stirred solution of pyrazolone $3(0.236 \mathrm{~g}, 0.001 \mathrm{~mol})$ in sodium acetate $(1 \mathrm{~g}$, dissolved in $5 \mathrm{~mL}$ of $25 \%$ aqueous ethanol). Stirring was continued for $4 \mathrm{~h}$ at $0-5{ }^{\circ} \mathrm{C}$ and the precipitated product separated upon dilution with cold water $(25 \mathrm{~mL})$ was filtered off, washed with water several times, dried and recrystallized from ethanol:benzene (1:1) (Scheme 2). Color: White. Yield: 80.2 \%. M.p.: $182-184{ }^{\circ} \mathrm{C}$. FT-IR (KBr, v, $\left.\mathrm{cm}^{-1}\right): 1712$ (C=0), $1663(\mathrm{C}=\mathrm{N}) .{ }^{1} \mathrm{H}$ NMR $\left(300 \mathrm{MHz}, \mathrm{CDCl}_{3}, \delta, \mathrm{ppm}\right): 8.23-8.24(\mathrm{~d}$, $1 \mathrm{H}, \mathrm{NCH}), 7.52-8.07(\mathrm{~m}, 4 \mathrm{H}, \mathrm{ArH}), 7.50-7.51(\mathrm{~d}, 1 \mathrm{H},=\mathrm{CH}-\mathrm{S})$, 7.04-7.25 (m, 6H, ArH), 3.87 (s, 1H, CH). MS (EI, $m / z$ (\%)): 347
(62.7, M+·), 236 (16.4), 129 (100), 84 (19.4), 78 (19.4), 77 (74.6), 55 (4.5). Anal. calcd. for $\mathrm{C}_{18} \mathrm{H}_{13} \mathrm{~N}_{5} \mathrm{OS}$ : C, 62.23; H, 3.77; N, 20.16; S, 9.23. Found: C, 62.41; H, 3.92, N, 20.19; S, $9.41 \%$.

\subsubsection{1,3-Diphenyl-4-(phenyldiazenyl)-1H-pyrazol-5-ol (18)}

Aniline $(0.093 \mathrm{~mL}, 0.001 \mathrm{~mol})$ in conc. $\mathrm{HCl}(3.6 \mathrm{~mL})$ and water $(1 \mathrm{~mL})$ was cooled in ice bath at $0-5{ }^{\circ} \mathrm{C}$ and diazotized with the solution of sodium nitrite $(0.345 \mathrm{~g})$ in water $(2 \mathrm{~mL})$. The cold diazonium solution was added slowly to a well stirred solution pyrazolone $3(0.236 \mathrm{~g}, 0.001 \mathrm{~mol})$ in pyridine $(20 \mathrm{~mL})$. The reaction mixture was stirred for another $2 \mathrm{~h}$. The crude product was filtered off, dried and recrystallized from ethanol (Scheme 2). Color: Orange. Yield: 55 \%. M.p.: $160-162{ }^{\circ} \mathrm{C}$. FT-IR $\left(\mathrm{KBr}, v, \mathrm{~cm}^{-1}\right): 3435(\mathrm{OH}), 1650(\mathrm{C}=0) .{ }^{1} \mathrm{H}$ NMR $(300 \mathrm{MHz}$, $\mathrm{CDCl}_{3}, \delta, \mathrm{ppm}$ ): 14.07 (s, $1 \mathrm{H}, \mathrm{OH} \mathrm{D} \mathrm{D}_{2} \mathrm{O}$ exchangeable), 7.22-8.28 (m, 15H, ArH). MS (EI, $m / z(\%)): 340$ (72.6, M+), 263 (26.0), 235 (28.3), 220 (6.0), 77 (100), 78 (7.5). Anal. calcd. for $\mathrm{C}_{21} \mathrm{H}_{16} \mathrm{~N}_{4} \mathrm{O}$ : C, 74.10; H, 4.74; N, 16.46. Found: C, 74.23; H, 4.91; N, $16.53 \%$.

\subsubsection{4-(4-Methoxybenzylidene)-1,3-diphenyl-1H-Pyrazol- 5(4H)-one (19)}

Method A: A mixture of pyrazolone 3 (2.36 g, $0.01 \mathrm{~mol}), p$ anisaldehyde $(1.36 \mathrm{~mL}, 0.01 \mathrm{~mol})$ in ethanol $(70 \mathrm{~mL})$ in presence of piperidine $(1 \mathrm{~mL})$ was refluxed for $2 \mathrm{~h}$. A white powder was formed during reflux. After cooling, the white powder was filtered off, washed with ethanol, dried and crystallized from ethanol (Scheme 2). Color: White. Yield: 81.6\%. M.p.: 218-220 ${ }^{\circ} \mathrm{C}$. FT-IR (KBr, v, $\left.\mathrm{cm}^{-1}\right): 3054(\mathrm{CH}$ aromatic), 2950 (CH aliphatic), 1714 (C=0). ${ }^{1} \mathrm{H}$ NMR $(300 \mathrm{MHz}$, DMSO, $\delta, \mathrm{ppm})$ : 6.76-8.04 (m, 14H, ArH), $5.13(\mathrm{~s}, 1 \mathrm{H}, \mathrm{CH}), 3.86$ $\left(\mathrm{s}, 3 \mathrm{H}, \mathrm{OCH}_{3}\right)$. 


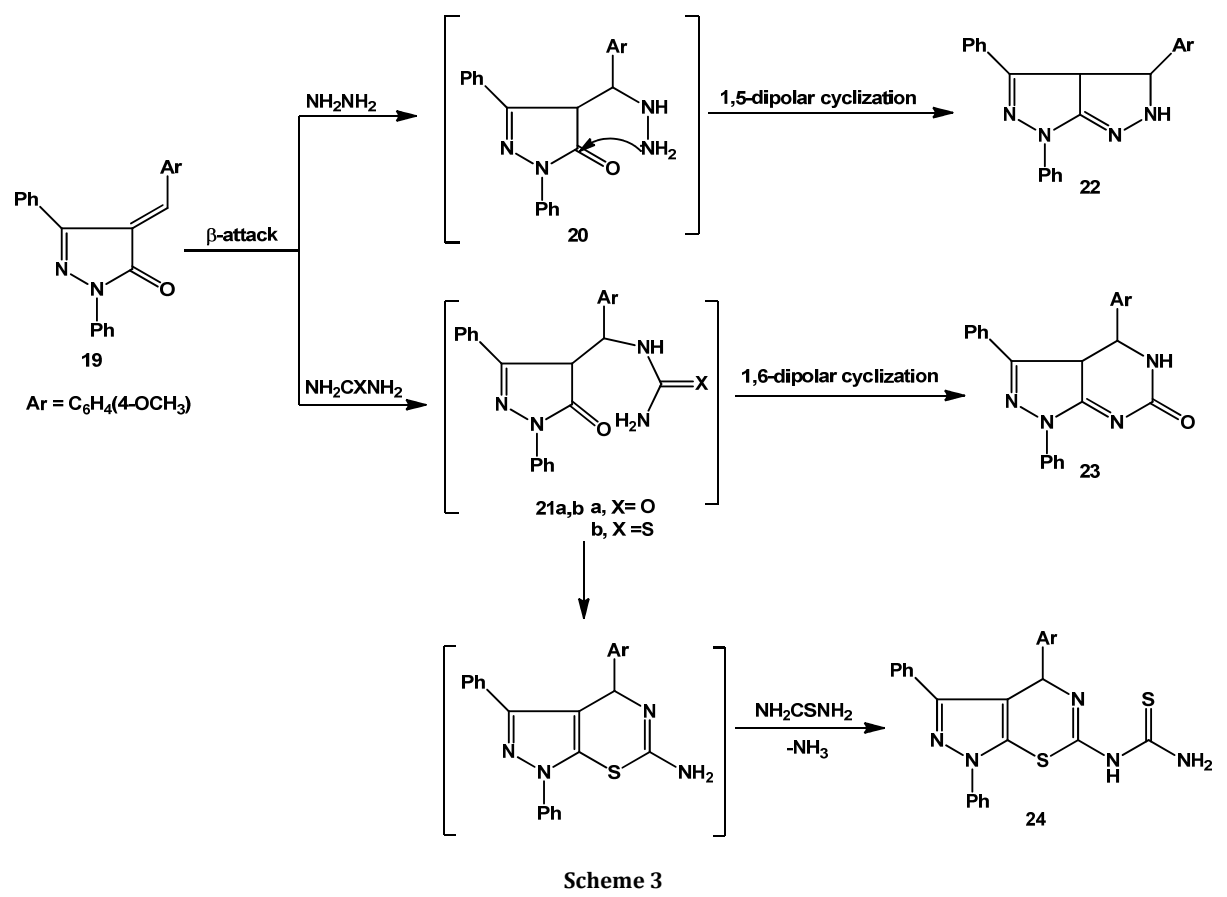

MS (EI, $m / z$ (\%)): 354 (71.7, M+·), 247 (36.1), 236 (42.8), 78 (8.6), 57 (13.9), 56(100). Anal. calcd. for $\mathrm{C}_{23} \mathrm{H}_{18} \mathrm{~N}_{2} \mathrm{O}_{2}$ : C, 77.95; H, 5.12; N, 7.90. Found: C, 78.19; H, 5.30; N, $8.13 \%$.

Method B: A mixture of pyrazolone $3(2.36 \mathrm{~g}, 0.01 \mathrm{~mol}), p$ anisaldehyde $(1.36 \mathrm{~mL}, 0.01 \mathrm{~mol})$ in presence of piperidine $(1 \mathrm{~mL})$ was heated under microwave irradiation for $2 \mathrm{~min}$ at power 180. After cooling, the separated solid was filtered off, washed with ethanol, dried and recrystallized from ethanol.

\subsubsection{4-(4-Methoxyphenyl)-1,3-diphenyl-1,3a,4,5- tetrahydropyrazolo[3,4-c]pyrazole (22)}

Method A: A mixture of pyrazolone $19(3.54 \mathrm{~g}, 0.01 \mathrm{~mol})$, hydrazine hydrate $(0.5 \mathrm{~mL}, 0.01 \mathrm{~mol})$ in ethanol $(30 \mathrm{~mL})$ was refluxed for $2 \mathrm{~h}$. the reaction mixture was then poured onto ice: $\mathrm{HCl}(1: 3)$, the solid product that formed was filtered off and washed with water $(4 \times 30 \mathrm{~mL})$, dried and recrystallized from benzene (Scheme 3). Color: Purple. Yield: 98\%. M.p.: 185-187 ${ }^{\circ} \mathrm{C}$. FT-IR (KBr, v, $\left.\mathrm{cm}^{-1}\right): 3623(\mathrm{NH}), 3056,3030$ (CH aromatic), $1569(\mathrm{C}=\mathrm{N}) .{ }^{1} \mathrm{H}$ NMR $(300 \mathrm{MHz}, \mathrm{DMSO}, \delta, \mathrm{ppm}): 8.12$ (s, $1 \mathrm{H}, \mathrm{NH}$, $\mathrm{D}_{2} \mathrm{O}$ exchangeable), 6.76-8.04 (m, $\left.14 \mathrm{H}, \mathrm{ArH}\right), 5.13(\mathrm{~s}, 1 \mathrm{H}$, $\mathrm{CHC}=\mathrm{N}), 3.82(\mathrm{~s}, 1 \mathrm{H}, \mathrm{CH}-\mathrm{NH}), 3.68\left(\mathrm{~s}, 3 \mathrm{H}, \mathrm{OCH}_{3}\right) . \mathrm{MS}(\mathrm{EI}, \mathrm{m} / \mathrm{z}$ (\%)): $370(0.5, \mathrm{M}+2), 355$ (24.1), 354 (100), 353 (30.2), 247 (47.5), 221 (33.2), 77 (44.8). Anal. calcd. for $\mathrm{C}_{23} \mathrm{H}_{20} \mathrm{~N}_{4} \mathrm{O}$ : C, 74.98; H, 5.47; N, 15.21. Found: C, 75.14; H, 5.62; N, $15.35 \%$.

Method B: A mixture of pyrazolone $19(3.54 \mathrm{~g}, 0.01 \mathrm{~mol})$, hydrazine hydrate $(0.5 \mathrm{~mL}, 0.01 \mathrm{~mol})$ was heated under microwave irradiation for $4 \mathrm{~min}$ at power 360 Watt. The separated solid was filtered off and washed with water $(4 \times$ $30 \mathrm{~mL}$ ), dried and recrystallized from benzene.

\subsubsection{4-(4-Methoxyphenyl)-1,3-diphenyl-4,5-dihydro-1H- pyrazolo[3,4-d]pyrimidin-6(3aH)-one (23)}

A mixture of pyrazolone 19 ( $3.54 \mathrm{~g}, 0.01 \mathrm{~mol})$, and urea $(0.6 \mathrm{~g}, 0.01 \mathrm{~mol})$ in glacial acetic acid $(20 \mathrm{~mL})$ was refluxed for $5 \mathrm{~h}$. The separated solid after pouring on crushed ice was filtered off, washed with water, dried and crystallized from benzene (Scheme 3). Color: Yellow. Yield: 45 \%. M.p.: 143-145 ${ }^{\circ} \mathrm{C}$. FT-IR (KBr, v, cm-1): $3625(\mathrm{NH}), 1723(\mathrm{C}=0), 1602(\mathrm{C}=\mathrm{N}) .{ }^{1} \mathrm{H}$ NMR (300 MHz, $\left.\mathrm{CDCl}_{3}, \delta, \mathrm{ppm}\right): 6.76-8.07(\mathrm{~m}, 14 \mathrm{H}, \mathrm{ArH}), 5.17$ (s, $1 \mathrm{H}, \mathrm{CHNH}), 3.91\left(\mathrm{~s}, 3 \mathrm{H}, \mathrm{OCH}_{3}\right), 3.78\left(\mathrm{~s}, 1 \mathrm{H}, \mathrm{NH} \mathrm{D}_{2} \mathrm{O}\right.$ exchangeable), 3.72 (s, 1H, CH). MS (EI, $m / z$ (\%)): 355 (12.4, [M-CHNO ]+), 247 (20.7), 236 (59.9), 77 (100), 78 (9.1). Anal. calcd. for $\mathrm{C}_{24} \mathrm{H}_{20} \mathrm{~N}_{4} \mathrm{O}_{2}$ : C, 72.71, H; 5.08, N; 14.13. Found: C, $72.90 ; \mathrm{H}, 5.21 ; \mathrm{N}, 14.31 \%$.

\subsubsection{1-(4-(4-Methoxyphenyl)-1,3-diphenyl-1,4- dihydropyrazolo[4,3-e][1,3]thiazin-6-yl)thiourea (24)}

A mixture of pyrazolone 19 (3.54 g, $0.01 \mathrm{~mol}$ ) and thiourea $(0.76 \mathrm{~g}, 0.01 \mathrm{~mol})$ in glacial acetic acid $(20 \mathrm{~mL})$ was refluxed for $5 \mathrm{~h}$. The reaction mixture was poured on crushed ice to give a yellow solid which was filtered off, washed with water, dried and crystallized from benzene:ethanol (1:1) (Scheme 3). Color: Yellow. Yield: $60.15 \%$. M.p.: $205-207^{\circ} \mathrm{C}$. FT-IR $\left(\mathrm{KBr}, v, \mathrm{~cm}^{-1}\right)$ : $3380,3276\left(\mathrm{NH}, \mathrm{NH}_{2}\right), 1614(\mathrm{C}=\mathrm{N}) .{ }^{1} \mathrm{H}$ NMR $\left(300 \mathrm{MHz}, \mathrm{CDCl}_{3}, \delta\right.$, ppm): 6.79-7.96 (m, 14H, ArH), 5.16 (s, 1H, benzilic), 3.69 (s, $\left.3 \mathrm{H}, \mathrm{OCH}_{3}\right), 3.08\left(\mathrm{~s}, 3 \mathrm{H}, \mathrm{NH}, \mathrm{NH}_{2} \mathrm{D}_{2} \mathrm{O}\right.$ exchangeable). MS (EI, $m / z$ (\%)): 471 (23.3, M+), 236 (26.7), 235 (30.0), 91 (30.0), 77 (100). Anal. calcd. for $\mathrm{C}_{25} \mathrm{H}_{21} \mathrm{~N}_{5} \mathrm{OS}_{2}$ : C, 63.67; $\mathrm{H}, 4.49 ; \mathrm{N}, 14.85 ; \mathrm{S}$, 13.60. Found: C, $63.82 ; \mathrm{H}, 4.60 ; \mathrm{N}, 14.93 ; \mathrm{S}, 13.81 \%$.

\subsubsection{3-(4-Methoxyphenyl)-4,6-diphenyl-3a,6-dihydro-3H- pyrazolo[3,4-c]isoxazole (25)}

A mixture of pyrazolone $19(3.54 \mathrm{~g}, 0.01 \mathrm{~mol})$ and hydroxylamine hydrochloride $(0.69 \mathrm{~g}, 0.01 \mathrm{~mol})$ in presence of pyridine $(10 \mathrm{~mL})$ was refluxed for $6 \mathrm{~h}$, the separated solid after cooling was filtered off, dried and crystallized from petroleum ether $80-100{ }^{\circ} \mathrm{C}$ (Scheme 4). Color: Pale yellow. Yield: $70.1 \%$. M.p.: $112-115{ }^{\circ} \mathrm{C}$. FT-IR ( $\left.\mathrm{KBr}, \mathrm{v}, \mathrm{cm}^{-1}\right): 3058$ (CH aromatic), 2952 (CH aliphatic), $1600(\mathrm{C}=\mathrm{N}) .{ }^{1} \mathrm{H}$ NMR $\left(300 \mathrm{MHz}, \mathrm{CDCl}_{3}, \delta\right.$, ppm): 6.74-8.55 (m, 14H, ArH), $3.91(\mathrm{~d}, 1 \mathrm{H}, \mathrm{CH}-0), 3.83(\mathrm{~s}, 3 \mathrm{H}$ $\left.\mathrm{OCH}_{3}\right), 2.85(\mathrm{~d}, 1 \mathrm{H}, \mathrm{CH}-\mathrm{C}=\mathrm{N})$. MS (EI, $\left.m / z(\%)\right): 354(42,[\mathrm{M}-$ $\left.\mathrm{CH}_{3} \mathrm{O}^{+}\right]^{+}$), 247 (17.4), 235 (49.9), 77 (100), 57 (3.5). Anal. calcd. for $\mathrm{C}_{23} \mathrm{H}_{19} \mathrm{~N}_{3} \mathrm{O}_{2}$ : C, 74.78; $\mathrm{H}, 5.18 ; \mathrm{N}, 11.37$. Found: $\mathrm{C}, 75.10 ; \mathrm{H}$, $5.25 ; \mathrm{N}, 11.56 \%$. 


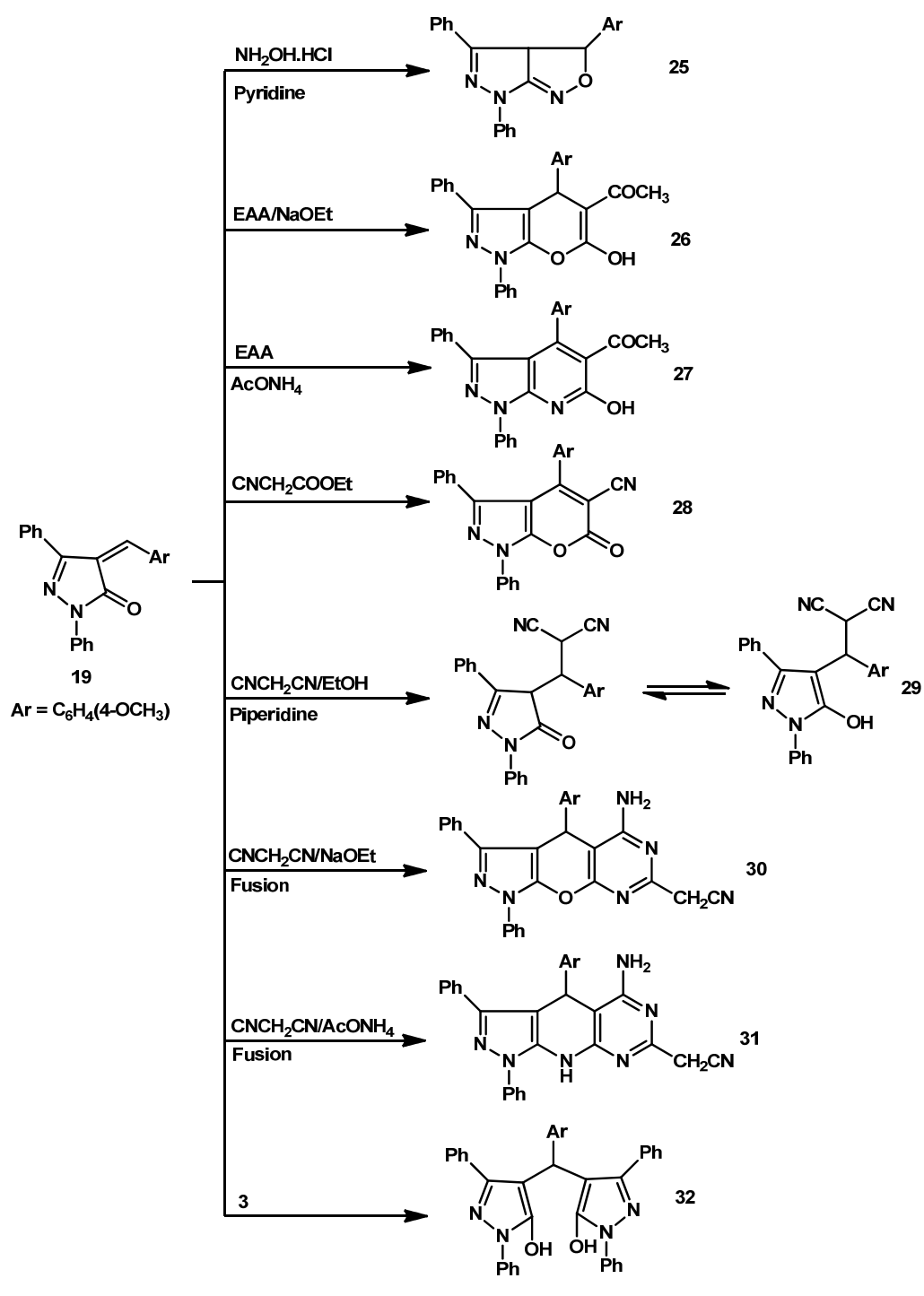

Scheme 4

2.2.20. 1-(6-Hydroxy-4-(4-methoxyphenyl)-1,3-diphenyl-1,4dihydropyrano[2,3-c]pyrazol-5-yl)ethanone (26)

A mixture of pyrazolone $19(0.708 \mathrm{~g}, 0.002 \mathrm{~mol})$, ethyl acetoacetate $(0.26 \mathrm{~mL}, 0.002 \mathrm{~mol}$, ) in sodium ethoxide $(1 \%)$ solution was refluxed for $6 \mathrm{~h}$. The mixture was poured onto ice: $\mathrm{HCl}(1: 3)$, the separated solid was filtered off, washed with water, dried and recrystallized from ethanol (Scheme 4). Color: Pale yellow. Yield: 73.17 \%. M.p.: $140-142{ }^{\circ} \mathrm{C}$. FT-IR $(\mathrm{KBr}, \mathrm{v}$, $\left.\mathrm{cm}^{-1}\right)$ : $3629(\mathrm{OH}), 1713(\mathrm{C}=0), 1601(\mathrm{C}=\mathrm{N}) .{ }^{1} \mathrm{H}$ NMR $(300 \mathrm{MHz}$, DMSO, $\delta, \mathrm{ppm}): 6.84-8.68(\mathrm{~m}, 14 \mathrm{H}, \mathrm{ArH}), 6.00\left(\mathrm{~s}, 1 \mathrm{H}, \mathrm{OH}, \mathrm{D}_{2} \mathrm{O}\right.$ exchangeable), $5.21\left(\mathrm{~s}, 1 \mathrm{H}\right.$, benzilic), $3.91\left(\mathrm{~s}, 3 \mathrm{H}, \mathrm{OCH}_{3}\right), 3.71(\mathrm{~s}$, $\left.3 \mathrm{H}, \mathrm{COCH}_{3}\right)$. MS (EI, $\left.m / z(\%)\right): 440\left(9.4,[\mathrm{M}+2 \cdot]^{+}\right), 354(71.8)$, 353 (34.1), 352 (21.2), 268 (100), 267 (45.9), 251 (28.2), 249 (58.8), 172 (15.3),77 (40.0), 78 (11.8). Anal. calcd. for $\mathrm{C}_{27} \mathrm{H}_{22} \mathrm{~N}_{2} \mathrm{O}_{4}$ : C, 73.96; H, 5.06; N, 6.39. Found: C, 74.14; H, 5.20; $\mathrm{N}, 6.51 \%$.

2.2.21. 1-(6-Hydroxy-4-(4-methoxyphenyl)-1,3-diphenyl-1Hpyrazolo[3,4-b]pyridin-5yl)ethanone (27)

A mixture of pyrazolone $19(0.708 \mathrm{~g}, 0.002 \mathrm{~mol})$, ammonium acetate $(2 \mathrm{~g})$, and ethyl acetoacetate $(0.26 \mathrm{~mL}$,
$0.002 \mathrm{~mol}$,) was fused for $7 \mathrm{~h}$ at $170{ }^{\circ} \mathrm{C}$. After cooling, the reaction mixture was triturated with petroleum ether $(60-80$ ${ }^{\circ} \mathrm{C}$ ) to give an orange solid which was recrystallized from petroleum ether $60-80{ }^{\circ} \mathrm{C}$ (Scheme 4). Color: Orange. Yield: 66.66\%. M.p.:136-138 ${ }^{\circ} \mathrm{C}$. FT-IR (KBr, v, cm $\left.{ }^{-1}\right): 3055(\mathrm{OH}) 1706$ $(\mathrm{C}=0) .{ }^{1} \mathrm{H}$ NMR $\left(300 \mathrm{MHz}, \mathrm{CDCl}_{3}, \delta, \mathrm{ppm}\right): 14.89$ (s, $1 \mathrm{H}, \mathrm{OH}, \mathrm{D}_{2} \mathrm{O}$ exchangeable), 7.12-7.91 (m, $14 \mathrm{H}, \mathrm{ArH}), 3.76\left(\mathrm{~s}, 3 \mathrm{H}, \mathrm{OCH}_{3}\right)$, 3.60 (s, 3H, $\left.\mathrm{COCH}_{3}\right)$. MS (EI, $\left.m / z(\%)\right): 435\left(3.4, \mathrm{M}^{+}\right), 289$ (2.9), 276 (9.1), 171 (7.4), 121 (100). Anal. calcd. for $\mathrm{C}_{27} \mathrm{H}_{21} \mathrm{~N}_{3} \mathrm{O}_{3}$ : C, 74.47; H, 4.86; N, 9.65. Found: C, 74.51; H, 4.93; N, $9.72 \%$.

\subsubsection{4-(4-Methoxyphenyl)-6-oxo-1,3-diphenyl-1,6-dihydro} pyrano[2,3-c]pyrazole-5-carbonitrile (28)

A mixture of pyrazolone $19(0.708 \mathrm{~g}, 0.002 \mathrm{~mol})$, ethyl cyanoacetate $(0.226 \mathrm{~mL}, 0.002 \mathrm{~mol})$ in sodium ethoxide $(1 \%)$ solution was refluxed for $6 \mathrm{~h}$. The mixture was poured onto ice:HCl (1:3), the separated solid was filtered off, washed with water, dried and recrystallized from ethanol (Scheme 4). Color: Pale green. Yield: $35.16 \%$. M.p.: $136-138{ }^{\circ} \mathrm{C}$. FT-IR (KBr, v, $\left.\mathrm{cm}^{-1}\right): 2208(\mathrm{C} \equiv \mathrm{N}), 1706(\mathrm{C}=0), 1665(\mathrm{C}=\mathrm{N}), 1621(\mathrm{C}=\mathrm{C}) .{ }^{1} \mathrm{H}$ NMR (300 MHz, DMSO, $\delta, \mathrm{ppm}):$ 7.25-7.82 (m, 14H, ArH), 3.79 (s, $\left.3 \mathrm{H}, \mathrm{OCH}_{3}\right)$. MS (EI, $\left.m / z(\%)\right): 354\left(9.4,\left[\mathrm{M}^{-} \mathrm{C}_{3} \mathrm{H}_{3} \mathrm{NO}^{-}\right]^{+}\right), 236$ 
(44.2), 105 (23.2), 91 (42.0), 85 (32.6), 69 (42.0), 57 (100), 51 (282). Anal. calcd. for $\mathrm{C}_{26} \mathrm{H}_{17} \mathrm{~N}_{3} \mathrm{O}_{3}$ : C, 74.45; $\mathrm{H}, 4.09$; N, 10.02 . Found: C, $74.62 ; \mathrm{H}, 4.13 ; \mathrm{N}, 10.21 \%$.

\subsubsection{2-((3-Methoxyphenyl)(5-oxo-1,3-diphenyl-4,5- dihydro-1H-pyrazol-4-yl)methyl)malononitrile (29)}

A mixture of pyrazolone $19(3.54 \mathrm{~g}, 0.01 \mathrm{~mol})$ and malononitrile $(0.66 \mathrm{~g}, 0.01 \mathrm{~mol})$ in ethanol $(30 \mathrm{~mL})$ in the presence of piperidine $(1 \mathrm{~mL})$ was refluxed for $10 \mathrm{~h}$. After cooling, solid crystals were produced which were filtered off, dried and recrystallized from ethanol:benzene (2:1) (Scheme 4). Color: Brown crystals. Yield: 69 \%. M.p.: 211-213 ${ }^{\circ} \mathrm{C}$. FT-IR $\left(\mathrm{KBr}, v, \mathrm{~cm}^{-1}\right): 3328(\mathrm{OH}), 2189,2153(\mathrm{C} \equiv \mathrm{N}), 1639(\mathrm{C}=0), 1601$ $(\mathrm{C}=\mathrm{N}) .{ }^{1} \mathrm{H}$ NMR $(300 \mathrm{MHz}, \mathrm{DMSO}, \delta, \mathrm{ppm}): 18.11\left(\mathrm{~s}, 1 \mathrm{H}, \mathrm{OH} \mathrm{D}_{2} \mathrm{O}\right.$ exchangeable), 6.76-8.03 (m, 14H, ArH), $5.12(\mathrm{~d}, 1 \mathrm{H}, \mathrm{CHCHCN})$, $3.68\left(\mathrm{~s}, 3 \mathrm{H}, \mathrm{OCH}_{3}\right), 3.15$ (d, $\left.1 \mathrm{H}, \mathrm{CHCHCN}\right)$. MS (EI, $\left.m / z(\%)\right): 420$ (3.1, M+), 236 (3.1), 84 (100), 70 (15.5), 57 (37.6). Anal. calcd. for $\mathrm{C}_{26} \mathrm{H}_{20} \mathrm{~N}_{4} \mathrm{O}_{2}$ : C, 74.27; $\mathrm{H}, 4.79 ; \mathrm{N}, 13.33$. Found: $\mathrm{C}, 73.95 ; \mathrm{H}$, $4.64 ; \mathrm{N}, 13.24 \%$.

\subsubsection{4. [5-Amino-4-(4-methoxyphenyl)-1,3-diphenyl-1,4- dihydro-9-oxa-1,2,6,8-tetraaza-cyclopenta[b]naphthalen-7- yl] acetonitrile (30)}

A mixture of pyrazolone $19(0.708 \mathrm{~g}, 0.002 \mathrm{~mol})$, malononitrile $(0.132 \mathrm{~g}, 0.002 \mathrm{~mol})$ in sodium ethoxide $(1 \%)$ solution was refluxed for $6 \mathrm{~h}$. The mixture was poured onto ice:hydrochloric acid, the solid that separated was filtered off, washed with water, dried and recrystallized from benzene (Scheme 4). Color: Pale yellow. Yield: 40 \%. M.p.: $117-119^{\circ} \mathrm{C}$. FT-IR (KBr, v, cm-1): 3424, $3322\left(\mathrm{NH}_{2}\right), 2213(\mathrm{C} \equiv \mathrm{N}), 1642$ $(\mathrm{C}=\mathrm{N}), 1602(\mathrm{C}=\mathrm{C}) .{ }^{1} \mathrm{H}$ NMR (300 MHz, DMSO, $\left.\delta, \mathrm{ppm}\right): 7.07-$ $7.83\left(\mathrm{~m}, 15 \mathrm{H}, 14 \mathrm{ArH}, 1 \mathrm{H}\right.$ benzilic), $6.00\left(\mathrm{~s}, 2 \mathrm{H}, \mathrm{NH}_{2} \mathrm{D}_{2} \mathrm{O}\right.$ exchangeable), $3.82\left(\mathrm{~s}, 2 \mathrm{H}, \mathrm{CH}_{2} \mathrm{CN}\right), 3.35\left(\mathrm{~s}, 3 \mathrm{H}, \mathrm{OCH}_{3}\right)$. MS (EI, $m / z(\%)): 443$ (54.5, [M-CHNO] ]), 295 (45.5), 138 (45.5), 122 (54.5), 109 (72.7), 108 (63.6), 93 (27.2), 67 (72.7)52 (100). Anal. calcd. for $\mathrm{C}_{29} \mathrm{H}_{22} \mathrm{~N}_{6} \mathrm{O}_{2}$ : C, 71.59; $\mathrm{H}, 4.56 ; \mathrm{N}, 17.27$. Found: $\mathrm{C}$, $71.61 ; \mathrm{H}, 4.71 ; \mathrm{N}, 17.41 \%$.

\subsubsection{5. [5-Amino-4-(4-methoxyphenyl)-1,3-diphenyl-4,9- dihydro-1H-pyrazolo [4',3':5,6]pyrido[2,3-d]pyrimidin-7- yl]acetonitrile (31)}

A mixture of pyrazolone $19(0.708 \mathrm{~g}, \quad 0.002 \mathrm{~mol})$, malononitrile $(0.132 \mathrm{~g}, 0.002 \mathrm{~mol})$ and ammonium acetate $(2 \mathrm{~g})$ was fused for $7 \mathrm{~h}$ at $170{ }^{\circ} \mathrm{C}$. After cooling, the solid was washed with water, filtered off, dried, and recrystallized from benzene:ethanol (1:2) (Scheme 4). Color: Pale yellow. Yield: 40 \%. M.p.: $190-192{ }^{\circ}$ C. FT-IR (KBr, v, cm$\left.{ }^{-1}\right): 3428,3321\left(\mathrm{NH}, \mathrm{NH}_{2}\right)$, $2203(\mathrm{C} \equiv \mathrm{N}), 1599(\mathrm{C}=\mathrm{C}) .{ }^{1} \mathrm{H}$ NMR $(300 \mathrm{MHz}, \mathrm{DMSO}, \delta, \mathrm{ppm})$ : 7.05-8.32 (m, 14H, ArH), 5.95 (s, 1H, benzilic), $5.70\left(\mathrm{~s}, 2 \mathrm{H}, \mathrm{NH}_{2}\right.$ $\mathrm{D}_{2} \mathrm{O}$ exchangeable), 5.66 (s, $1 \mathrm{H}, \mathrm{NH} \mathrm{D}_{2} \mathrm{O}$ exchangeable), $3.84(\mathrm{~s}$, $3 \mathrm{H}, \mathrm{OCH}_{3}$ ), 3.67 (s, 2H, CH $2 \mathrm{CN}$ ). MS (EI, $\left.m / z(\%)\right): 435$ (10.6, [M$\left.\mathrm{C}_{2} \mathrm{H}_{4} \mathrm{~N}_{2}\right]^{+}$), 435 (10.6), 236 (36.2), 160 (12.8), 93 (51.1), 84 (36.2), 78 (29.8), 77 (100), 56 (55.3). Anal. calcd. for $\mathrm{C}_{29} \mathrm{H}_{23} \mathrm{~N}_{7} \mathrm{O}$ : C, $71.74 ; \mathrm{H}, 4.77 ; \mathrm{N}, 20.19$. Found: C, 71.82; H, 4.93; N, $20.23 \%$.

\subsubsection{4-((5-Hydroxy-1,3diphenyl-1H-pyrazol-4-yl)(4- methoxyphenyl)methyl)-1,3-diphenyl-1H-pyrazol-5-ol (32)}

Pyrazolone $19(3.54 \mathrm{~g}, 0.01 \mathrm{~mol})$ was added at room temperature with stirring to a mixture of pyrazolone $3(2.36 \mathrm{~g}$, $0.01 \mathrm{~mol})$, and sodium ethoxide $(0.5 \mathrm{~g}$ sodium in $10 \mathrm{~mL})$, then the mixture was stirred for another $1 \mathrm{~h}$ at $100{ }^{\circ} \mathrm{C}$. The mixture was poured onto ice:hydrochloric acid (1:3), the separated solid was filtered off, washed with water, dried and recrystallized from ethanol (Scheme 4). Color: Red. Yield: 90.4 \%. M.p.:178-180 ${ }^{\circ} \mathrm{C}$. FT-IR (KBr, v, cm-1): $3448(\mathrm{OH}), 3058(\mathrm{CH}$ aromatic), 2928 (CH aliphatic), $1603(\mathrm{C}=\mathrm{N}) .{ }^{1} \mathrm{H}$ NMR $(300 \mathrm{MHz}$, DMSO, $\delta, \mathrm{ppm}): 8.62\left(\mathrm{~s}, 2 \mathrm{H}, \mathrm{OH} \mathrm{D}_{2} \mathrm{O}\right.$ exchangeable), 6.85-7.89 (m, 24H, ArH), 5.23 (s, 1H, benzilic), 3.89 (s, 3H, OCH $)$. MS (EI, $\mathrm{m} / \mathrm{z}(\%)): 590\left(0.01, \mathrm{M}^{+}\right), 236$ (60.6), 149 (100), 148 (33.3), 135 (12.1), 121 (21.2), 92 (16.7), 77 (45.5). Anal. calcd. for $\mathrm{C}_{38} \mathrm{H}_{30} \mathrm{~N}_{4} \mathrm{O}_{3}$ : C, 77.27; $\mathrm{H}, 5.12 ; \mathrm{N}, 9.49$. Found: $\mathrm{C}, 77.32 ; \mathrm{H}, 5.27$; $\mathrm{N}, 9.62 \%$.

\subsection{Antimicrobial activities}

The synthesized compounds were evaluated for their antimicrobial activities using the agar diffusion technique [16]. All Chemical compounds were dissolved in DMF (5000 ppm).

The tested compounds were evaluated against Gram -ve bacteria (Pseudomonas aeruginosa ATCC 10145 and Escherichia coli ATCC 23282), Gram +ve bacteria (Bacillus subtilis ATCC 6633, Micrococcus luteus ATCC 25922) and Yeast (Candida albicans IMRU 3669 and Filamentous niger ATCC 16404). The bacteria and yeast were grown on nutrient agar while the fungus was grown on Czapek's Dox agar medium.

The negative control DMF was showed no antimicrobial activity against the tested microorganism, and the positive control was Erythromycin for bacteria and Metronidazole for yeast and fungus.

All examination were done in duplicates and the listed are the average of the obtained results.

\section{Results and discussion}

\subsection{Synthesis}

The starting material pyrazolone $\mathbf{3}$ was prepared by literature known procedure [17] using ethyl benzoyl acetate 1 as $\beta$-keto ester and phenyl hydrazine, 2 . In principle, for compounds unsubstituted at pyrazole $\mathrm{C}-4$, three isomers are possible, assigned as 1H-pyrazol-5-ol (A), 2,4-dihydro- $3 H$ pyrazol-3-one (B) and 1,2-dihydro-3H-pyrazol-3-one (C) (Scheme 5).
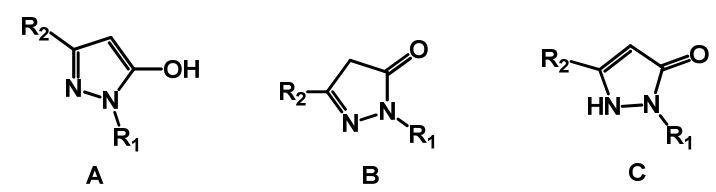

Scheme 5

The IR spectra of 3 displayed band at $1706 \mathrm{~cm}^{-1}$ due to $\mathrm{C}=0$ absorption at position 5 and the ${ }^{1} \mathrm{H}$ NMR spectrum devoid of exchangeable $\mathrm{NH}$ or $\mathrm{OH}$ which indicated that pyrazolone 3 exists in the keto form in either the solid state or the liquid state.

The reactivity of hydrogen atoms at C- 4 is the most outstanding chemical property of pyrazolone 3 towards condensation reactions (Scheme 1).

Amino carbonitriles $\mathbf{4}$ and $\mathbf{5}$ and pyrazolopyridine $\mathbf{6}$ were synthesized via a multicomponent reaction of pyrazolone $\mathbf{3}$ with $p$-anisaldehyde and active methylene compounds such as malononitrile and/or ethyl acetoacetate in the presence of ammonium acetate and/or piperidine at $140-170{ }^{\circ} \mathrm{C}$. The appearance of the cyano and the amino functions in the IR of the reaction products support the structures of compounds $\mathbf{4}$, and $\mathbf{5 .}$

It has been reported that amino carbonitriles reacted with ammonium thiocyanate [18] in boiling acetic acid to give (thioxo pyrimidinyl) thiourea derivatives, while in our investigation we isolated the amino(iminomethyl)thiocyanato methanethioimidate 7 through two consecutive additions of two molecules of ammonium thiocyanate to compound $\mathbf{4}$. The structure of compound $\mathbf{7}$ was confirmed by IR which showed band at $2207(\mathrm{C} \equiv \mathrm{N})$, and also its reaction with hydrazine hydrate to afford pyrazolo[3,4-b]pyridin-6-amine derivative 8 . 
Table 1. Microwave synthesis of compounds 5, 19 and 22.

\begin{tabular}{llll}
\hline Compound no & Power (Watt) & Time (min) & Yield (\%) MW/Conventional \\
\hline 5 & 180 & 4 & $50 / 14$ \\
19 & 180 & 2 & $90 / 82$ \\
22 & 360 & 4 & $99 / 98$ \\
\hline
\end{tabular}

Table 2. The Antimicrobial activities of compounds 8, 23, 27 and $31 *$

\begin{tabular}{|c|c|c|c|c|c|c|}
\hline $\begin{array}{l}\text { Compound } \\
\text { no }\end{array}$ & Bacillus subtilis & Micrococcus lutes & Pseudonomus aerugino & Escherichia coli & Candida albicans & Filamentous niger \\
\hline 8 & - & - & - & - & - & - \\
\hline 23 & - & - & 17.0 & 20.0 & - & - \\
\hline 27 & 36.0 & 27.0 & 27.0 & 32.0 & 21.0 & 17.0 \\
\hline 31 & 23.0 & 21.0 & 18.0 & 22.0 & 20.0 & 15.0 \\
\hline Erythromycin & 32.0 & 32.0 & 30.0 & 44.0 & - & - \\
\hline Metronidazole & - & - & - & - & 27.0 & 25.0 \\
\hline DMF & - & - & - & - & - & - \\
\hline
\end{tabular}

* Erythromycin was used as a standard against Gram positive and Gram negative bacteria, while metronidazole was used as a standard against fungi, and the negative controle was DMF.

Treatment of compound $\mathbf{4}$ with formamide in the presence of formic acid and DMF afforded the open structure pyrazolo[3,4-b]pyridinylformamide 9 .

Acetylation of compounds $\mathbf{4}$ and $\mathbf{5}$ with acetic anhydride afforded the acetylated compounds pyrazolo[3,4-b]pyridin-6yl)acetamide (10) and dihydropyrano[2,3-c]-pyrazol-6yl)acetamide 11, respectively. The structures of these compounds were confirmed by spectroscopic data and also the reaction of compound $\mathbf{1 1}$ with hydrazine hydrate to give the bis compound 12. The hydrazine hydrate is an ambident nucleophile therefore one molecule of it attacks two molecules of compound $\mathbf{1 1}$ followed by hydrogenation to afford the bis compound $\mathbf{1 2}$ (Scheme 1).

Condensation of pyrazolone $\mathbf{3}$ with glucose in ethanol/DMF/drops of $\mathrm{AcOH}$ mixture furnished the penta hydroxy hexylidene pyrazolone derivative $\mathbf{1 3}$.

The active methylene group of pyrazolone 3 reacted with phenyl isothiocyanate [19] in the presence of piperidine to give an intermediate thiolate anion $\mathbf{1 4}$ which upon treatment with dilute hydrochloric acid furnished functionalized thioamide $\mathbf{1 5}$ (Scheme 2).

Compound $\mathbf{1 5}$ is a versatile multifunctional reagent which reacted with hydrazine hydrate to afford pyrazolo[3,4c]pyrazolethione 16.

Novel 4-thiazolyl diazenyl- $1 H$-pyrazol-5-one (17) and 4-(phenyldiazenyl)-1H-pyrazol-5-ol (18) have been obtained as red and orange dyes respectively via the diazonium cation resulting from the interaction of nitrite with pyrazolone $\mathbf{3}$ followed by coupling with 2 -aminothiazole and aniline (Scheme 2).

The pyrazolone 3 was reacted with p-anisaldehyde [17] in the presence of piperidine to give the pyrazolone 19 according to Knoevenagel condensation reaction which is an important tool for the formation of new carbon-carbon bonds.

To show the synthetic potentiality of compound 19, the reactivity of compound 19 with different nitrogen nucleophiles such as hydrazine hydrate, urea, thiourea and hydroxylamine hydrochloride was examined (Scheme 3,4).

Reactions of compound 19 with hydrazine hydrate and urea afforded pyrazolo[3,4-c]pyrazole (22) and pyrazolo[3,4d]pyrimidinone (23) via $\beta$-attack on the $\mathrm{C}=\mathrm{C}$ moiety in compound 19 to give the non-isolable intermediates 20, 21a, which underwent 1,5 and 1,6 intramolecular dipolar cyclization.

Reaction of thiourea with compound 19 via $\beta$-attack on the $\mathrm{C}=\mathrm{C}$ moiety in compound $\mathbf{1 9}$ gave the non-isolable intermediate 21b which underwent 1,6 intramolecular dipolar cyclization through the sulfur atom, followed by the loss of ammonia through the reaction with another molecule of thiourea to give pyrazolo[4,3-e][1,3] thiazinyl thiourea (24).
Hydroxylamine hydrochloride was reacted with compound 19 in the presence of pyridine to give pyrazolo[3,4-c]isoxazole (25).

In this work, we aim to study the reactivity of compound $\mathbf{1 9}$ as $\alpha, \beta$-unsaturated compound towards several carbon nucleophiles under Michael reaction (Scheme 4).

The formation of pyrano[2,3-c] pyrazolyl ethanone (26) and pyrazolo[3,4-b]pyridinyl ethanone (27) seems to proceed by the addition of the nucleophile ethyl acetoacetate on the $\beta$ carbon of the electrophile (19) in a conjugate addition reaction in the presence of sodium ethoxide and/or ammonium acetate followed by 6-exo-dig cyclization and dehydrogenation. Similarly compound $\mathbf{2 8}$ is formed by the addition of ethyl cyanoacetate to pyrazolone $\mathbf{3}$ in the presence of sodium ethoxide.

The addition of malononitrile to compound 19 in the presence of piperidine afforded the adduct compound 29 which exists in the keto/enol forms which was inferred from its spectroscopic data. IR spectrum showed bands at $1639 \mathrm{~cm}^{-1}$ for $v_{\mathrm{C}=0}$, and $3328 \mathrm{~cm}^{-1}$ due to $v_{\mathrm{OH}},{ }^{1} \mathrm{H}$ NMR spectrum showed $\delta$ $18.11 \mathrm{ppm}$ for $\mathrm{OH}$.

On the other hand, the addition of malononitrile to compound 19 in the presence of sodium ethoxide and/or ammonium acetate afforded compounds $\mathbf{3 0}$ and $\mathbf{3 1}$. Malononitirile was added to the $\beta$-carbon of compound 19 followed by nucleophilic addition of the hydroxyl and/or the amino groups to the cyano group through 6-exo-dig cyclization, then another molecule of malononitrile was attacked by the formed amino group followed by cyclization and hydrogen transfer to give compounds $\mathbf{3 0}$ and $\mathbf{3 1}$, respectively.

Stirring of compound 19 with pyrazolone (3) in the presence of sodium ethoxide afforded hydroxy pyrazolyl pyrazolol (32).

It was observed that microwave irradiation method was practically superior to conventional heating method in terms of higher yields, rapid, and environmentally benign process. We carried out some of the reactions using microwave irradiation to improve the yield and the time of the reactions. Synthesis of amino carbonitriles (5), pyrazolone (19) and pyrazolo[3,4c]pyrazole (22) under microwave irradiation showed improvement in the yield and the time of the reactions (Table 1). Formation of compounds 5, 19 and 22 under microwave irradiation produced the products in good yield within 2-4 minutes. The reactions were carried out under solvent free conditions to avoid problems associated with solvent use such as cost, handling and specifically safety, because of fire hazard due to occurrence of sparks in microwave ovens. The reactions were less effective and took two hours to be completed when carried out in ethanol under reflux.

\subsection{Antimicrobial activities}


Compound $\mathbf{2 7}$ showed higher reactivity towards Bacillus subtilis than the reference and high reactivity towards Gram -ve bacteria, Gram +ve bacteria and fungi. Compound $\mathbf{3 1}$ showed moderate reactivity towards Gram -ve bacteria, Gram +ve bacteria and fungi. Compound 8 showed no reactivity towards Gram -ve bacteria, Gram +ve bacteria and fungi (Table 2).

\section{Conclusion}

Pyrazolone $\mathbf{3}$ is an active compound towards condensation reactions. Pyrazolone 19 acts as an $\alpha, \beta$-unsaturated compound towards different carbon and nitrogen nucleophiles. Microwave irradiation method was practically superior to conventional heating method in terms of higher yields, rapid, and environmentally benign process.

\section{Acknowledgements}

Technical support from Department of Chemistry, Faculty of Science, Ain Shams University is gratefully acknowledged.

\section{References}

[1]. Kumar, L.; Thakur, C.; Sharma, V. Int. J. Res. Pharm. Sci. 2012, 2, 13-22

[2]. Halen, P. K.; Murumkar, P.; Giridhar, R.; Yadav, M. R. Mini-Rev. Med. Chem. 2009, 9, 124-139.

[3]. Kucukguzel, G.; Rollas, S.; Erdeniz, H.; Kiraz, M.; Ekinci, A.; Cevdet, V. A. Eur. J. Med. Chem. 2000, 35, 761-771.

[4]. Mariappan, G.; Saha, B. P.; Sutharson, L.; Ankit, Garg, S. Pandey, L. Kumar, D. J. Pharm. Res. 2010, 3, 2856-2859.

[5]. Devnath, H. P.; Islam, M. R. Bangladesh J. Pharmacol. 2010, 5, 30-33

[6]. Rahat, K.; Imam, U.; Alam, M.; Sultan, D. Bangladesh J. Pharmacol. 2008, 3, 27-35.

[7]. Soni, J. P.; Sen, D. J.; Modh, K. M. J. Appl. Pharm. Sci. 2011, 1, 115-120.

[8]. Sunitha, S.; Aravindakshan, K. K. Int. J. Pharm. Bio. Sci. 2011, 2, 108113.

[9]. Vijesh, A. M.; Arun, M. I.; Shrikrishna, I.; Shivananda, K. N.; Shyma, P. C.; Arulmoli, T. Der Pharma Chem. 2011, 3, 454-463.

[10]. Kumar, K. S.; Rajasekharan, A. Int. J. Res. Pharm. Chem. 2012, 2, $327-$ 337.

[11]. Evstropov, A. N.; Yavorovskaya, V. E.; Vorobev, E. S.; Khudonogova, Z. P.; Medvedeva, S. G.; Filimonov, V. D.; Prishchep, T. P.; Saratikov, A. S. Pharm. Chem. J. 1992, 26, 426-430.

[12]. Nirupam, D.; Abilasha, V.; Shrivastava, K.; Shrivastva, P.; Sushant, K. Indian J. Chem. B 2008, 47, 1555-1558.

[13]. Amir, M.; Shikha, K. Indian J. Chem. B 2005, 44, 2532-2537.

[14]. Ragab, F. A.; Abdel-Gawad, N. M.; Georgey, H. H.; Said, M. F. Chem. Pharm. Bull. 2013, 61(8), 834-845.

[15]. Al-Mutairi, A. A.; El-Baih, F. E. M.; Al-Hazimi, H. M. J. Saudi Chem. Soc. 2010, 14, 287-299.

[16]. Cooper, R. E.; Kavanageh, F. W. Analytical Microbiology, Vol. I and II, Academic press: New York and London, 1972.

[17]. Vogel, A. I. Elementary Practical Organic Chemistry Part II, Qualitative Organic Analysis, 2 ${ }^{\text {nd }}$ edition, Longman, London 1966.

[18]. Abdel-Fattah, A. M.; Sherif, S. M.; El-Reedy, A. M.; Gad-Alla, S. A. Phosphorus Sulfur 1992, 70, 67-73.

[19]. Khalil, M. A.; Sayed, S. M.; Raslan, M. A. Am. J. Org. Chem. 2012, 2, 161170 . 